\title{
Some Classes of Difference Sequence Spaces of Fuzzy Real Numbers Defined by Orlicz Function
}

\author{
Binod Chandra Tripathy ${ }^{1}$ and Stuti Borgohain ${ }^{2}$ \\ ${ }^{1}$ Mathematical Sciences Division, Institute of Advanced Study in Science and Technology, Paschim Boragaon, Garchuk, \\ Guwahati 781035, India \\ ${ }^{2}$ Department of Mathematics, Indian Institute of Technology Bombay, Powai, Maharashtra, Mumbai 400076, India
}

Correspondence should be addressed to Binod Chandra Tripathy, tripathybc@rediffmail.com

Received 12 August 2011; Accepted 10 November 2011

Academic Editor: Ajith Abraham

Copyright (C) 2011 B. C. Tripathy and S. Borgohain. This is an open access article distributed under the Creative Commons Attribution License, which permits unrestricted use, distribution, and reproduction in any medium, provided the original work is properly cited.

We introduce the classes of generalized difference bounded, convergent, and null sequences of fuzzy real numbers defined by an Orlicz function. Some properties of these sequence spaces like solidness, symmetricity, and convergence-free are studied. We obtain some inclusion relations involving these sequence spaces.

\section{Introduction}

The concept of fuzzy set theory was introduced by Zadeh in 1965. Later on sequences of fuzzy numbers have been discussed by Syau [1], Tripathy and Baruah [2], Tripathy and Borgohain [3], Tripathy and Dutta $[4,5]$, Tripathy and Sarma $[6,7]$, and many others.

Kizmaz [8] defined the difference sequence spaces $\ell_{\infty}(\Delta)$, $c(\Delta)$, and $c_{0}(\Delta)$ of complex numbers as follows:

$$
Z(\Delta)=\left\{x=\left(x_{k}\right):\left(\Delta x_{k}\right) \in Z\right\}, \quad \text { for } Z=\ell_{\infty}, c, c_{0},
$$

where $\Delta x=\left(\Delta x_{k}\right)=\left(x_{k}-x_{k+1}\right)$.

The above spaces are Banach spaces, normed by

$$
\|x\|_{\Delta}=\left|x_{1}\right|+\sup _{k}\left|\Delta x_{k}\right|
$$

The idea of Kizmaz [8] was applied to introduce different type of difference sequence spaces and study their different properties by Et et al. [9], Tripathy et al. [10], Tripathy and Baruah [2], Tripathy and Borgohain [3], Tripathy and Esi [11], Tripathy et al. [12], Tripathy and Mahanta [13], and many others.
Tripathy and Esi [11] introduced a new type of difference sequence spaces as follows. Let $m \in N$ be fixed, then

$$
\begin{aligned}
& Z\left(\Delta_{m}\right)=\left\{x=\left(x_{k}\right):\left(\Delta_{m} x_{k}\right) \in Z\right\}, \quad \text { for } Z=\ell_{\infty}, c, c_{0}, \\
& \text { where } \Delta_{m} x=\left(\Delta_{m} x_{k}\right)=\left(x_{k}-x_{k+m}\right) .
\end{aligned}
$$

The above sequence spaces are Banach spaces, normed by

$$
\|x\|_{\Delta}=\sum_{r=1}^{m}\left|x_{r}\right|+\sup _{k}\left|\Delta_{m} x_{k}\right|
$$

Tripathy et al. [12] further generalized this notion and introduced the following. For $m \geq 1$ and $n \geq 1$,

$$
\begin{aligned}
& Z\left(\Delta_{m}^{n}\right)=\left\{x=\left(x_{k}\right):\left(\Delta_{m}^{n} x_{k}\right) \in Z\right\}, \quad \text { for } Z=\ell_{\infty}, c, c_{0}, \\
& \text { where } \Delta_{m}^{n} x_{k}=\Delta_{m}^{n-1} x_{k}-\Delta_{m}^{n-1} x_{k+m}, \quad \forall k \in N .
\end{aligned}
$$

This generalized difference has the following binomial representation:

$$
\Delta_{m}^{n} x_{k}=\sum_{r=0}^{n}(-1)^{r}\left(\begin{array}{l}
n \\
r
\end{array}\right) x_{k+r m} .
$$


An Orlicz function is a function $M:[0, \infty) \rightarrow[0, \infty)$, which is continuous, nondecreasing, and convex with $M(0)=0, M(x)>0$, for $x>0$ and $M(x) \rightarrow \infty$, as $x \rightarrow \infty$ (one may refer to Korasnoselkii and Rutitsky [14]).

An Orlicz function $M$ is said to satisfy $\Delta_{2}$-condition for all values of $x$, if there exists a constant $K>0$, such that $M(L x) \leq K L M(x)$, for all $x>0$ and for $L>1$.

Remark 1. An Orlicz function satisfies the inequality $M(\lambda x) \leq$ $\lambda M(x)$, for all $\lambda$ with $0<\lambda<1$.

Throughout the paper $w^{F}, \ell^{F}, \ell_{\infty}^{F}$ represent the classes of all, absolutely summable, and bounded sequences of fuzzy real numbers, respectively.

\section{Definitions and Background}

Let $C\left(R^{n}\right)=\left\{A \subset R^{n}: A\right.$ is compact and convex $\}$. Then the space $C\left(R^{n}\right)$ has linear structure induced by the operations $A+B=\{a+b: a \in A, b \in B\}$ and $\lambda A=\{\lambda a: a \in A\}$ for $A, B \in C\left(R^{n}\right)$ and $\lambda \in R$.

The Hausdorff distance between $A$ and $B$ of $C\left(R^{n}\right)$ is defined as

$$
\delta_{\infty}(A, B)=\max \left\{\sup _{a \in A} \inf _{b \in B}\|a-b\|, \sup _{b \in B} \inf _{a \in A}\|a-b\|\right\} .
$$

It is well known that $\left(C\left(R^{n}\right), \delta_{\infty}\right)$ is a complete metric space. A fuzzy real number on $R^{n}$ is a function $X: R^{n} \rightarrow$ $I(=[0,1])$ associating each real number $t \in R^{n}$ with its grade of membership $X(t)$.

A fuzzy real number $X$ is called convex if $X(t) \geq X(s) \wedge$ $X(r)=\min (X(s), X(r))$, where $s<t<r$.

If there exists $t_{0} \in R^{n}$ such that $X\left(t_{0}\right)=1$, then the fuzzy real number $X$ is called normal.

A fuzzy real number $X$ is said to be upper semicontinuous if for each $\varepsilon>0, X^{-1}([0, a+\varepsilon))$, for all $a \in I$ is open in the usual topology of $R^{n}$.

The class of all upper semi-continuous, normal, convex fuzzy real numbers is denoted by $R^{n}(I)$. Let $X \in R^{n}(I)$, then the $\alpha$-level set $X^{\alpha}$, for $0<\alpha \leq 1$, is defined by, $X^{\alpha}=\{t \in$ $\left.R^{n}: X(t) \geq \alpha\right\}$ and is a nonempty compact convex subset of $R^{n}$. The 0-level set, that is, $X^{0}$, is the closure of strong 0 -cut, that is, $X^{0}=c l\left\{t \in R^{n}: X(t)>0\right\}$. The absolute value of $X \in R(I)$, that is, $|X|$, is defined by

$$
|X|(t)= \begin{cases}\max \{X(t), X(-t)\}, & \text { for } t \geq 0, \\ 0, & \text { otherwise. }\end{cases}
$$

For $r \in R^{n}, \bar{r} \in R^{n}(I)$ is defined as,

$$
\bar{r}(t)= \begin{cases}1, & \text { for } t=r \\ 0, & \text { otherwise. }\end{cases}
$$

The additive identity and multiplicative identity of $R^{n}(I)$ are denoted by $\bar{\theta}$ and $\bar{e}$, respectively, where $\theta=(0,0, \ldots, 0)$ and $e=(1,1, \ldots, 1)$. The zero sequence of fuzzy real numbers is denoted by $\bar{\vartheta}=\{\bar{\theta}, \bar{\theta}, \ldots, \bar{\theta}, \ldots\}$.
The linear structure of $C\left(R^{n}\right)$ induces the addition $X+Y$ and scalar multiplication $\lambda X, \lambda \in R$ in terms of $\alpha$-level sets, by $[X+Y]^{\alpha}=[X]^{\alpha}+[Y]^{\alpha}$ and $[\lambda X]^{\alpha}=\lambda[X]^{\alpha}$, for each $0<\alpha \leq 1$, where

$$
\lambda X(t)= \begin{cases}\overline{0}, & \text { for } \lambda=0 \\ X\left(\lambda^{-1} t\right), & \text { otherwise }\end{cases}
$$

Define, for each $1 \leq q<\infty$,

$$
d_{q}(X, Y)=\left(\int_{0}^{1} \delta_{\infty}\left(X^{\alpha}, Y^{\alpha}\right)^{q} d \alpha\right)^{1 / q}
$$

and $d_{\infty}: R^{n}(I) \times R^{n}(I) \rightarrow R$ such that $d_{\infty}(X, Y)=$ $\sup _{0<\alpha \leq 1} \delta_{\infty}\left(X^{\alpha}, Y^{\alpha}\right)$, where $\delta_{\infty}$ is the Hausdorff metric. Clearly, $d_{\infty}(X, Y)=\lim _{q \rightarrow \infty} d_{q}(X, Y)$ with $d_{q} \leq d_{r}$ if $q \leq r$. Moreover $\left(R^{n}(I), d_{\infty}\right)$ is a complete, separable, and locally compact metric space.

A sequence $X=\left(X_{k}\right)$ of fuzzy real numbers is said to converge to the fuzzy number $X_{0}$, if for every $\varepsilon>0$, there exists $k_{0} \in N$ such that $d_{\infty}\left(X_{k}, X_{0}\right)<\varepsilon$, for all $k \geq k_{0}$.

A sequence space $E$ is said to be solid if $\left(Y_{n}\right) \in E$, whenever $\left(X_{n}\right) \in E$ and $\left|Y_{n}\right| \leq\left|X_{n}\right|$, for all $n \in N$.

Let $X=\left(X_{n}\right)$ be a sequence, then $S(X)$ denotes the set of all permutations of the elements of $\left(X_{n}\right)$, that is, $S(X)=$ $\left\{\left(X_{\pi(n)}\right): \pi\right.$ is a permutation of $\left.N\right\}$. A sequence space $E$ is said to be symmetric if $S(X) \subset E$ for all $X \in E$.

A sequence space $E$ is said to be convergence-free if $\left(Y_{n}\right) \in$ $E$ whenever $\left(X_{n}\right) \in E$ and $X_{n}=\bar{\theta}$ implies $Y_{n}=\bar{\theta}$.

A sequence space $E$ is said to be monotone if $E$ contains the canonical preimages of all its step spaces.

Lemma 2. A class of sequences $E$ is solid which implies that $E$ is monotone.

Lindenstrauss and Tzafriri [15] used the notion of Orlicz function and introduced the sequence space:

$$
\ell_{M}=\left\{\left(x_{k}\right) \in w: \sum_{k=1}^{\infty} M\left(\frac{\left|x_{k}\right|}{\rho}\right)<\infty \text {, for some } \rho>0\right\} \text {. }
$$

The space $\ell_{M}$ with the norm,

$$
\left\|\left(x_{k}\right)\right\|=\inf \left\{\rho>0: \sum_{k=1}^{\infty} M\left(\frac{\left|x_{k}\right|}{\rho}\right) \leq 1\right\},
$$

becomes a Banach space, which is called an Orlicz sequence space. The space $\ell_{M}$ is closely related to the space $\ell_{p}$, which is an Orlicz sequence space with $M(x)=x^{p}$, for $1 \leq p<\infty$.

In the later stage different classes of Orlicz sequence spaces were introduced and investigated by Altin et al. [16], Et et al. [9], Tripathy et al. [10], Tripathy and Borgohain [3], Tripathy and Hazarika [17], Tripathy and Mahanta [13], Tripathy and Sarma $[6,7,18]$, and many others. 
In this paper we introduce the following difference sequence spaces:

$$
\begin{aligned}
& \ell_{\infty}^{F}\left(M, \Delta_{m}^{n}\right)=\left\{\left(X_{k}\right) \in w^{F}: \sup _{k}\left(\frac{d_{\infty}\left(\Delta_{m}^{n} X_{k}, \bar{\theta}\right)}{\rho}\right)<\infty,\right. \\
&\text { for some } \rho>0\} \\
& c^{F}\left(M, \Delta_{m}^{n}\right)=\left\{\left(X_{k}\right) \in w^{F}: \lim _{k \rightarrow \infty}\left(\frac{d_{\infty}\left(\Delta_{m}^{n} X_{k}, L\right)}{\rho}\right)=0,\right.\text { for some } \left.\rho>0, L \in R^{n}(I)\right\} \\
& c_{0}^{F}\left(M, \Delta_{m}^{n}\right)=\left\{\left(X_{k}\right) \in w^{F}: \lim _{k \rightarrow \infty}\left(\frac{d_{\infty}\left(\Delta_{m}^{n} X_{k}, \bar{\theta}\right)}{\rho}\right)=0,\right. \\
&\text { for some } \rho>0\} .
\end{aligned}
$$

\section{Main Results}

Theorem 3. The classes of sequences $\ell_{\infty}^{F}\left(M, \Delta_{m}^{n}\right), c^{F}\left(M, \Delta_{m}^{n}\right)$, $c_{0}^{F}\left(M, \Delta_{m}^{n}\right)$ are complete metric spaces by the metric

$$
\begin{aligned}
\eta(X, Y)= & \sum_{r=1}^{m n} d_{\infty}\left(X_{r}, Y_{r}\right) \\
& +\inf \left\{\rho>0: \sup _{k} M\left(\frac{d_{\infty}\left(\Delta_{m}^{n} X_{k}, \Delta_{m}^{n} Y_{k}\right)}{\rho}\right) \leq 1\right\},
\end{aligned}
$$

for $X, Y \in \ell_{\infty}\left(M, \Delta_{m}^{n}\right)^{F}, c^{F}\left(M, \Delta_{m}^{n}\right), c_{0}^{F}\left(M, \Delta_{m}^{n}\right)$.

Proof. We establish the result for the class of sequences $\ell_{\infty}^{F}\left(M, \Delta_{m}^{n}\right)$. The proof for the other cases will follow similarly. It can easily be verified that $\ell_{\infty}^{F}\left(M, \Delta_{m}^{n}\right)$ is a metric space by the metric $\eta$ defined above. Next we show that it is a complete metric space.

Let $\left(X^{(i)}\right)$ be a Cauchy sequence in $\ell_{\infty}^{F}\left(M, \Delta_{m}^{n}\right)$ such that $X^{(i)}=\left(X_{n}^{(i)}\right)_{n=1}^{\infty}$. Let $\varepsilon>0$ be given. For a fixed $x_{0}>0$, choose $r>0$ such that $M\left(r x_{0} / 2\right) \geq 1$. Then there exits a positive integer $n_{0}=n_{0}(\varepsilon)$ such that

$$
\eta\left(X^{(i)}, X^{(j)}\right)<\frac{\varepsilon}{r x_{0}}, \quad \forall i, j \geq n_{0} .
$$

By the definition of $\eta$, we have,

$$
\begin{array}{r}
\sum_{r=1}^{m n} d_{\infty}\left(X_{r}{ }^{(i)}, X_{r}{ }^{(j)}\right) \\
\quad+\inf \left\{\rho>0: \sup _{k} M\left(\frac{d_{\infty}\left(\Delta_{m}^{n} X_{r}^{(i)}, \Delta_{m}^{n} X_{r}^{(j)}\right)}{\rho}\right) \leq 1\right\} \leq \varepsilon, \\
\forall i, j \geq n_{0},
\end{array}
$$

which implies

$$
\begin{aligned}
& \sum_{r=1}^{m n} d_{\infty}\left(X_{r}^{(i)}, X_{r}^{(j)}\right)<\varepsilon, \quad \forall i, j \geq n_{0}, \\
& \Longrightarrow d_{\infty}\left(X_{r}^{(i)}, X_{r}^{(j)}\right)<\varepsilon, \quad \forall i, j \geq n_{0}, r=1,2,3, \ldots, m n .
\end{aligned}
$$

Hence $\left(X_{r}^{(i)}\right)$, for $r=1,2,3, \ldots, m n$ are Cauchy sequence in $R^{n}(I)$ and hence are convergent in $R^{n}(I)$, since $R^{n}(I)$ is a complete metric space.

Let

$$
\lim _{i \rightarrow \infty} X_{r}^{(i)}=X_{r}, \quad \text { for } r=1,2,3, \ldots, m n .
$$

Also,

$$
\begin{array}{r}
\sup _{k} M\left(\frac{d_{\infty}\left(\Delta_{m}^{n} X_{k}^{(i)}, \Delta_{m}^{n} X_{k}^{(j)}\right)}{\rho}\right) \leq 1, \quad \forall i, j \geq n_{0}, \\
\Longrightarrow M\left(\frac{d_{\infty}\left(\Delta_{m}^{n} X_{k}^{(i)}, \Delta_{m}^{n} X_{k}^{(j)}\right)}{\eta\left(X^{(i)}, X^{(j)}\right)}\right) \leq 1 \leq M\left(\frac{r x_{0}}{2}\right), \\
\forall i, j \geq n_{0} .
\end{array}
$$

Since $M$ is continuous, we get,

$$
\begin{aligned}
& d_{\infty}\left(\Delta_{m}^{n} X_{k}^{(i)}, \Delta_{m}^{n} X_{k}^{(j)}\right) \leq \frac{r x_{0}}{2} \cdot \eta\left(X^{(i)}, X^{(j)}\right), \quad \forall i, j \geq n_{0}, \\
& \Longrightarrow d_{\infty}\left(\Delta_{m}^{n} X_{k}^{(i)}, \Delta_{m}^{n} X_{k}^{(j)}\right)<\frac{r x_{0}}{2} \cdot \frac{\varepsilon}{r x_{0}}=\frac{\varepsilon}{2}, \quad \forall i, j \geq n_{0}, \\
& \Longrightarrow d_{\infty}\left(\Delta_{m}^{n} X_{k}^{(i)}, \Delta_{m}^{n} X_{k}^{(j)}\right)<\frac{\varepsilon}{2}, \quad \forall i, j \geq n_{0},
\end{aligned}
$$

which implies $\left(\Delta_{m}^{n} X_{k}^{(i)}\right)$ is a Cauchy sequence in $R^{n}(I)$ and so is convergent in $R^{n}(I)$, since $R^{n}(I)$ is complete metric space.

Let $\lim _{i} \Delta_{m}^{n} X_{k}^{(i)}=Y_{k}$ (say), in $R^{n}(I)$, for each $k \in N$.

We have to prove that

$$
\lim _{i} X^{(i)}=X, \quad X \in \ell_{\infty}^{F}\left(M, \Delta_{m}^{n}\right) .
$$

For $k=1$, we have, from (6) and (19),

$$
\lim _{i \rightarrow \infty} X_{m n+1}^{(i)}=X_{m n+1}, \quad \text { for } m \geq 1, n \geq 1 .
$$


Proceeding in this way inductively, we get

$$
\lim _{i \rightarrow \infty} X_{k}^{(i)}=X_{k}, \quad \text { for each } k \in N \text {. }
$$

Also, $\lim _{i} \Delta_{m}^{n} X_{k}^{(i)}=\Delta_{m}^{n} X_{k}$, for each $k \in N$.

Next taking $j \rightarrow \infty$, keeping $i$ fixed, and by the continuity of $M$, we have the following from (20):

$$
\sup _{k} M\left(\frac{d_{\infty}\left(\Delta_{m}^{n} X_{k}^{(i)}, \Delta_{m}^{n} X_{k}\right)}{\rho}\right) \leq 1, \quad \text { for some } \rho>0 .
$$

Now on taking the infimum of such $\rho$ 's, we get

$$
\begin{array}{r}
\inf \left\{\rho>0: \sup _{k} M\left(\frac{d_{\infty}\left(\Delta_{m}^{n} X_{k}^{(i)}, \Delta_{m}^{n} X_{k}\right)}{\rho}\right) \leq 1\right\}<\varepsilon, \\
\forall i \geq n_{0}(\operatorname{by}(2)) .
\end{array}
$$

Hence from (17) on taking limit as $j \rightarrow \infty$, we get

$$
\begin{array}{r}
\sum_{r=1}^{m n} d_{\infty}\left(X_{r}^{(i)}, X_{r}\right) \\
+\inf \left\{\rho>0: \sup _{k} M\left(\frac{d_{\infty}\left(\Delta_{m}^{n} X_{k}^{(i)}, \Delta_{m}^{n} X_{k}\right)}{\rho}\right) \leq 1\right\} \\
<\varepsilon+\varepsilon=2 \varepsilon, \quad \forall i \geq n_{0},
\end{array}
$$

which implies

$$
\eta\left(X^{(i)}, X\right)<2 \varepsilon, \quad \forall i \geq n_{0}
$$

That is, $\lim _{i} X^{(i)}=X$.

Next we show that $X \in \ell_{\infty}^{F}\left(M, \Delta_{m}^{n}\right)$.

We know that

$$
d_{\infty}\left(\Delta_{m}^{n} X_{k}, \bar{\theta}\right) \leq d_{\infty}\left(\Delta_{m}^{n} X_{k}^{(i)}, \Delta_{m}^{n} X_{k}\right)+d_{\infty}\left(\Delta_{m}^{n} X_{k}^{(i)}, \bar{\theta}\right) .
$$

Since $M$ is continuous and nondecreasing, so we get

$$
\begin{aligned}
\sup _{k} M\left(\frac{d_{\infty}\left(\Delta_{m}^{n} X_{k}, \bar{\theta}\right)}{\rho}\right) \leq & \sup _{k} M\left(\frac{d_{\infty}\left(\Delta_{m}^{n} X_{k}^{(i)}, \Delta_{m}^{n} X_{k}\right)}{\rho}\right) \\
& +\sup _{k} M\left(\frac{d_{\infty}\left(\Delta_{m}^{n} X_{k}^{(i)}, \bar{\theta}\right)}{\rho}\right)<\infty
\end{aligned}
$$

which implies $X \in \ell_{\infty}^{F}\left(M, \Delta_{m}^{n}\right)$.

Hence $\ell_{\infty}^{F}\left(M, \Delta_{m}^{n}\right)$ is a complete metric space.

The other cases can be established similarly.

This completes the proof of the theorem.

Result 1. The classes of sequences $\ell_{\infty}^{F}\left(M, \triangle_{m}^{n}\right), c^{F}\left(M, \triangle_{m}^{n}\right)$, $c_{0}^{F}\left(M, \triangle_{m}^{n}\right)$, are neither solid nor monotone in general.
Proof. The result follows from the following example.

Example 4. Consider the sequence space $\ell_{\infty}^{F}\left(M, \Delta_{m}^{n}\right)$. Let $m=$ 2 and $n=3$. Let $M(x)=|x|$, for all $x \in[0, \infty)$.

Consider the sequence $\left(X_{k}\right)$ defined by

$$
X_{k}(t)= \begin{cases}1, & \text { for } k \in N, t=(k, k, k, \ldots), \\ 0, & \text { otherwise. }\end{cases}
$$

Then,

$$
\Delta_{2}^{3} X_{k}(t)= \begin{cases}1, & \text { for } k \in N, t=(0,0,0, \ldots), \\ 0, & \text { otherwise. }\end{cases}
$$

Then, we have $d_{\infty}\left(\Delta_{2}^{3} X_{k}, \bar{\theta}\right)=0$, for all $k \in N$.

Hence, we have

$$
\sup _{k} M\left(\frac{d_{\infty}\left(\Delta_{2}^{3} X_{k}, \bar{\theta}\right)}{\rho}\right)<\infty, \text { for some } \rho>0,
$$

which implies $\left(X_{k}\right) \in \ell_{\infty}^{F}\left(M, \Delta_{2}^{3}\right)$.

Consider the sequence $\left(\alpha_{k}\right)$ of scalars defined by

$$
\alpha_{k}= \begin{cases}1, & \text { for } k=i^{2}, i \in N, \\ 0, & \text { otherwise. }\end{cases}
$$

For $k=i^{2}$, we have

$$
\alpha_{k} X_{k}(t)= \begin{cases}1, & t=(k, k, k, \ldots), \\ 0, & \text { otherwise }\end{cases}
$$

For $k \neq i^{2}$, we have

$$
\alpha_{k} X_{k}(t)= \begin{cases}1, & t=(0,0,0, \ldots) \\ 0, & \text { otherwise }\end{cases}
$$

which implies

$$
\sup _{k} M\left(\frac{d_{\infty}\left(\Delta_{2}^{3} X_{k}, \bar{\theta}\right)}{\rho}\right)=\infty, \text { for each } \rho>0 .
$$

Hence $\left(\alpha_{k} X_{k}\right) \notin \ell_{\infty}^{F}\left(M, \Delta_{2}^{3}\right)$.

Thus, $\ell_{\infty}^{F}\left(M, \triangle_{m}^{n}\right)$ is not solid in general.

Similarly the other cases can be established. The classes of sequences are not monotone followed by Lemma 2.

Result 2. The classes of sequences $\ell_{\infty}^{F}\left(M, \triangle_{m}^{n}\right), c^{F}\left(M, \triangle_{m}^{n}\right)$, and $c_{0}^{F}\left(M, \triangle_{m}^{n}\right)$ are not symmetric in general.

Proof. The result follows from the following example.

Example 5. Let $m=2$ and $n=1$. Let $M(x)=x^{2}$, for all $x \in[0, \infty)$. Consider the sequence $\left(X_{k}\right)$ defined by

$$
X_{k}(t)= \begin{cases}1, & \text { for } k \in N, t=(k, k, k, \ldots), \\ 0, & \text { otherwise. }\end{cases}
$$


Then,

$\Delta_{2} X_{k}(t)= \begin{cases}1, & \text { for } k \in N, t=(-2,-2,-2, \ldots), \\ 0, & \text { otherwise. }\end{cases}$

Then, $d_{\infty}\left(\Delta_{2} X_{k}, \overline{0}\right)=1$, for all $k \in N$, which shows $\left(X_{k}\right) \in c^{F}\left(M, \Delta_{2}\right) \subset \ell_{\infty}^{F}\left(M, \Delta_{2}\right)$.

Let $\left(Y_{k}\right)$ be a rearrangement of $\left(X_{k}\right)$ such that $\left(Y_{k}\right)=\left(X_{1}, X_{2}, X_{4}, X_{3}, X_{9}, X_{5}, X_{16}, X_{6}, X_{25}, \ldots\right)$. Then we get $d_{\infty}\left(\Delta_{2} Y_{k}, \overline{0}\right) \approx k-(k-1)^{2} \approx k^{2}$, for all $k \in N$, which implies $\sup _{k} M\left(\frac{d_{\infty}\left(\Delta_{2} Y_{k}, \overline{0}\right)}{\rho}\right)=\infty, \quad$ for each fixed $\rho>0$.

Hence, $\left(Y_{k}\right) \notin \ell_{\infty}^{F}\left(M, \Delta_{2}\right)$.

Thus the classes of sequences $\ell_{\infty}^{F}\left(M, \Delta_{m}^{n}\right), c^{F}\left(M, \Delta_{m}^{n}\right)$, and $c_{0}^{F}\left(M, \Delta_{m}^{n}\right)$ are not symmetric in general.

Note 1. For $m=n=0$, the class of sequences $\ell_{\infty}^{F}(M)$ and $c^{F}(M)$ are symmetric. For $m \leq 1$ and $n \leq 1$, the class of sequences $c_{0}^{F}\left(M, \Delta_{m}^{n}\right)$ is symmetric.

Proposition 6. The classes of sequences $\ell_{\infty}^{F}\left(M, \Delta_{m}^{n}\right), c^{F}(M$, $\left.\Delta_{m}^{n}\right), c_{0}^{F}\left(M, \Delta_{m}^{n}\right)$ are not convergence-free in general.

Proof. The result follows from the following example.

Example 7. Let $m=4$ and $n=1$. Let $M(x)=x^{3}$, for all $x \in[0, \infty)$. Consider the sequence $\left(X_{k}\right)$ defined by

$$
X_{k}(t)= \begin{cases}1, & \text { for } k \in N, t=\left(\frac{1}{k}, \frac{1}{k}, \frac{1}{k}, \ldots\right), \\ 0, & \text { otherwise. }\end{cases}
$$

Then,

\section{$\Delta_{4} X_{k}(t)$}

$= \begin{cases}1, & \text { for } k \in N, t=\left(\frac{4}{k(k+4)}, \frac{4}{k(k+4)}, \frac{4}{k(k+4)}, \ldots\right), \\ 0, & \text { otherwise. }\end{cases}$

Hence we have $d_{\infty}\left(\Delta_{4} X_{k}, \bar{\theta}\right)=4 / k(k+4)$, which implies $\left(X_{n}\right) \in c_{0}^{F}\left(M, \Delta_{4}\right) \subset c^{F}\left(M, \Delta_{4}\right) \subset \ell_{\infty}^{F}\left(M, \Delta_{4}\right)$.

Consider the sequence $\left(Y_{k}\right)$ defined by

$$
Y_{k}(t)= \begin{cases}1, & \text { for } k \in N, t=\left(k^{2}, k^{2}, k^{2}, \ldots\right), \\ 0, & \text { otherwise }\end{cases}
$$

so that

$$
\begin{aligned}
& \Delta_{4} Y_{k}(t) \\
& = \begin{cases}1, & \text { for } k \in N, \\
& t=(-(8 k+16),-(8 k+16), \ldots), \\
0, & \text { otherwise. }\end{cases}
\end{aligned}
$$

Thus $d_{\infty}\left(\Delta_{4} Y_{k}, \bar{\theta}\right)=8 k+16$, for all $k \in N$, which implies

$$
\sup _{k} M\left(\frac{d_{\infty}\left(\Delta_{4} Y_{k}, \bar{\theta}\right)}{\rho}\right)=\infty, \text { for some } \rho>0 \text {. }
$$

Thus $\left(Y_{k}\right) \notin \ell_{\infty}^{F}\left(M, \Delta_{4}\right)$.

Hence the classes of sequences $\ell_{\infty}^{F}\left(M, \Delta_{m}^{n}\right), c^{F}\left(M, \Delta_{m}^{n}\right)$, $c_{0}^{F}\left(M, \Delta_{m}^{n}\right)$ are not convergence-free in general.

Theorem 8. Let $M, M_{1}$, and $M_{2}$ be Orlicz functions satisfying $\Delta_{2}$-condition. Then, for $Z=\ell_{\infty}^{F}, c^{F}$, and $c_{0}^{F}$,

(i) $Z\left(M_{1}, \Delta_{m}^{n}\right) \subseteq Z\left(M^{\circ} M_{1}, \Delta_{m}^{n}\right)$,

(ii) $Z\left(M_{1}, \Delta_{m}^{n}\right) \cap Z\left(M_{2}, \Delta_{m}^{n}\right) \subseteq Z\left(M_{1}+M_{2}, \Delta_{m}^{n}\right)$.

Proof. (i) Let $\left(X_{k}\right) \in Z\left(M_{1}, \Delta_{m}^{n}\right)$. Consider $\varepsilon>0$ and $\eta>0$ such that $\varepsilon=M(\eta)$.

Then,

$$
M_{1}\left(\frac{d_{\infty}\left(\Delta_{m}^{n} X_{k}, L\right)}{\rho}\right)<\eta, \text { for some } \rho>0 .
$$

Let

$$
Y_{k}=M_{1}\left(\frac{d_{\infty}\left(\Delta_{m}^{n} X_{k}, L\right)}{\rho}\right), \text { for some } \rho>0 .
$$

Since $M$ is continuous and non-decreasing, we get

$$
M\left(Y_{k}\right)=M\left(M_{1}\left(\frac{d_{\infty}\left(\Delta_{m}^{n} X_{k}, L\right)}{\rho}\right)\right)<M(\eta)=\varepsilon,
$$

for some $\rho>0$,

which implies $\left(X_{k}\right) \in Z\left(M^{\circ} M_{1}, \Delta_{m}^{n}\right)$.

This completes the proof.

(ii) Let $\left(X_{k}\right) \in Z\left(M_{1}, \Delta_{m}^{n}\right) \cap Z\left(M_{2}, \Delta_{m}^{n}\right)$.

Then,

$$
\begin{aligned}
& M_{1}\left(\frac{d_{\infty}\left(\Delta_{m}^{n} X_{k}, L\right)}{\rho}\right)<\varepsilon, \text { for some } \rho>0, \\
& M_{2}\left(\frac{d_{\infty}\left(\Delta_{m}^{n} X_{k}, L\right)}{\rho}\right)<\varepsilon, \text { for some } \rho>0 .
\end{aligned}
$$

The proof follows from the equality

$$
\begin{aligned}
& \left(M_{1}+M_{2}\right)\left(\frac{d_{\infty}\left(\Delta_{m}^{n} X_{k}, L\right)}{\rho}\right) \\
& =M_{1}\left(\frac{d_{\infty}\left(\Delta_{m}^{n} X_{k}, L\right)}{\rho}\right)+M_{2}\left(\frac{d_{\infty}\left(\Delta_{m}^{n} X_{k}, L\right)}{\rho}\right), \\
& <\varepsilon+\varepsilon=2 \varepsilon, \quad \text { for some } \rho>0
\end{aligned}
$$

which implies that $\left(X_{k}\right) \in Z\left(M_{1}+M_{2}, \Delta_{m}^{n}\right)$.

This completes the proof.

Proposition 9. One has $Z\left(M, \Delta_{m}^{i}\right) \subset Z\left(M, \Delta_{m}^{n}\right)$, for $0 \leq i<$ $n$, for $Z=\ell_{\infty}^{F}, c^{F}$, and $c_{0}^{F}$. 
Proof. Let $\left(X_{k}\right) \in \ell_{\infty}^{F}\left(M, \Delta_{m}^{n-1}\right)$. Then we have,

$$
\sup _{k \geq 1} M\left(\frac{d_{\infty}\left(\Delta_{m}^{n-1} X_{k}, \bar{\theta}\right)}{\rho}\right)<\infty .
$$

Now we have

$$
\begin{aligned}
\sup _{k \geq 1}( & \left(\frac{d_{\infty}\left(\Delta_{m}^{n} X_{k}, \bar{\theta}\right)}{\rho}\right) \\
= & \sup _{k \geq 1}\left(\frac{d_{\infty}\left(\Delta_{m}^{n-1} X_{k}-\Delta_{m}^{n-1} X_{k+m}, \bar{\theta}\right)}{\rho}\right) \\
\leq & \frac{1}{2} \sup _{k \geq 1}\left(\frac{d_{\infty}\left(\Delta_{m}^{n-1} X_{k}, \bar{\theta}\right)}{\rho}\right) \\
& +\frac{1}{2} \sup _{k \geq 1}\left(\frac{d_{\infty}\left(\Delta_{m}^{n-1} X_{k+m}, \bar{\theta}\right)}{\rho}\right)<\infty .
\end{aligned}
$$

Proceeding in this way, we have $Z\left(M, \Delta_{m}^{i}\right) \subset Z\left(M, \Delta_{m}^{n}\right)$, for $0 \leq i<n$, for $Z=\ell_{\infty}^{F}, c^{F}$, and $c_{0}^{F}$.

This completes the proof.

\section{References}

[1] Y. R. Syau, "Sequences in a fuzzy metric space," Computers and Mathematics with Applications, vol. 33, no. 6, pp. 73-76, 1997.

[2] B. C. Tripathy and A. Baruah, "New type of difference sequence spaces of fuzzy real numbers," Mathematical Modelling and Analysis, vol. 14, no. 3, pp. 391-397, 2009.

[3] B. C. Tripathy and S. Borgohain, "The sequence space $m(M$, $\left.\varnothing, \triangle^{n}{ }_{m}, P\right)^{F}, "$ Mathematical Modelling and Analysis, vol. 13, no. 4, pp. 577-586, 2008.

[4] B. C. Tripathy and A. J. Dutta, "On fuzzy real-valued double sequence space ${ }_{2} \ell_{F}^{p}$," Mathematical and Computer Modelling, vol. 46, no. 9-10, pp. 1294-1299, 2007.

[5] B. C. Tripathy and A. J. Dutta, "Bounded variation double sequence space of fuzzy real numbers," Computers and Mathematics with Applications, vol. 59, no. 2, pp. 1031-1037, 2010.

[6] B. C. Tripathy and B. Sarma, "Sequence spaces of fuzzy real numbers defined by Orlicz functions," Mathematica Slovaca, vol. 58, no. 5, pp. 621-628, 2008.

[7] B. C. Tripathy and B. Sarma, "Double sequence spaces of fuzzy numbers defined by Orlicz function," Acta Mathematica Scientia, vol. 31, no. 1, pp. 134-140, 2011.

[8] H. Kizmaz, "On certain sequence spaces," Canadian Mathematical Bulletin, vol. 24, no. 2, pp. 169-176, 1981.

[9] M. Et, Y. Altin, B. Choudhary, and B. C. Tripathy, "On some classes of sequences defined by sequences of orlicz functions," Mathematical Inequalities and Applications, vol. 9, no. 2, pp. 335-342, 2006.

[10] B. C. Tripathy, Y. Altin, and M. Et, "Generalized difference sequence spaces on seminormed space defined by Orlicz functions," Mathematica Slovaca, vol. 58, no. 3, pp. 315-324, 2008.

[11] B. C. Tripathy and A. Esi, "A new type of difference sequence spaces," International Journal of Science and Technology, vol. 1, no. 1, pp. 11-14, 2006.

[12] B. C. Tripathy, A. Esi, and B. K. Tipathy, "On a new type of generalized difference Cesàro sequence spaces," Soochow Journal of Mathematics, vol. 31, no. 3, pp. 333-340, 2005.
[13] B. C. Tripathy and S. Mahanta, "On a class of generalized lacunary difference sequence spaces defined by Orlicz functions," Acta Mathematicae Applicatae Sinica, vol. 20, no. 2, pp. 231238, 2004.

[14] M. A. Korasnoselkii and Y. B. Rutitsky, Convex Functions and Orlicz Functions, P. Noordhoff, Groningen, The Netherlands, 1961.

[15] J. Lindenstrauss and L. Tzafriri, "On orlicz sequence spaces," Israel Journal of Mathematics, vol. 10, no. 3, pp. 379-390, 1971.

[16] Y. Altin, M. Et, and B. C. Tripathy, "The sequence space $\left|\bar{N}_{P}\right|(M, r, q, s)$ on seminormed spaces," Applied Mathematics and Computation, vol. 154, no. 2, pp. 423-430, 2004.

[17] B. C. Tripathy and B. Hazarika, "Some I-convergent sequence spaces defined by Orlicz functions," Acta Mathematicae Applicatae Sinica, vol. 27, no. 1, pp. 149-154, 2011.

[18] B. C. Tripathy and B. Sarma, "Vector valued double sequence spaces defined by orlicz function," Mathematica Slovaca, vol. 59, no. 6, pp. 767-776, 2009. 

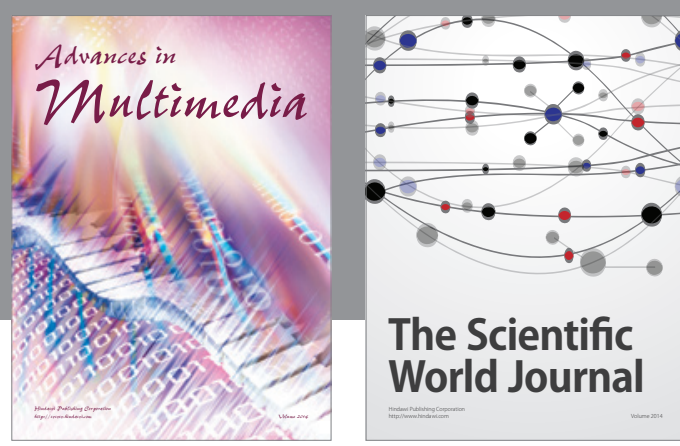

The Scientific World Journal
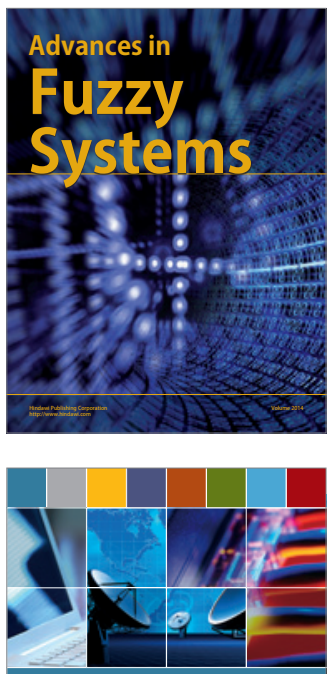

Computer Networks and Communications
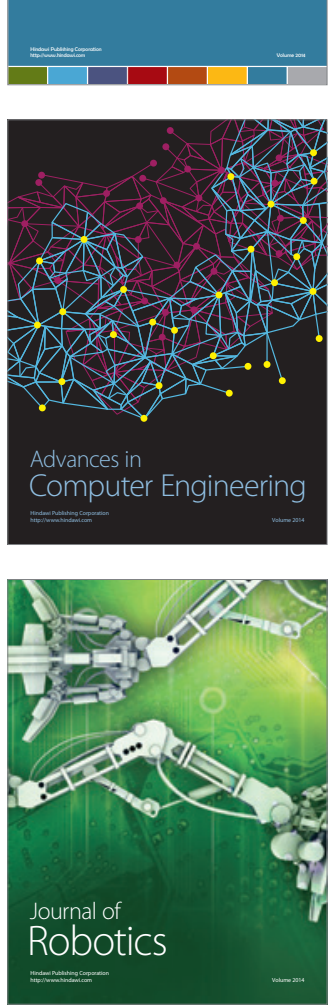
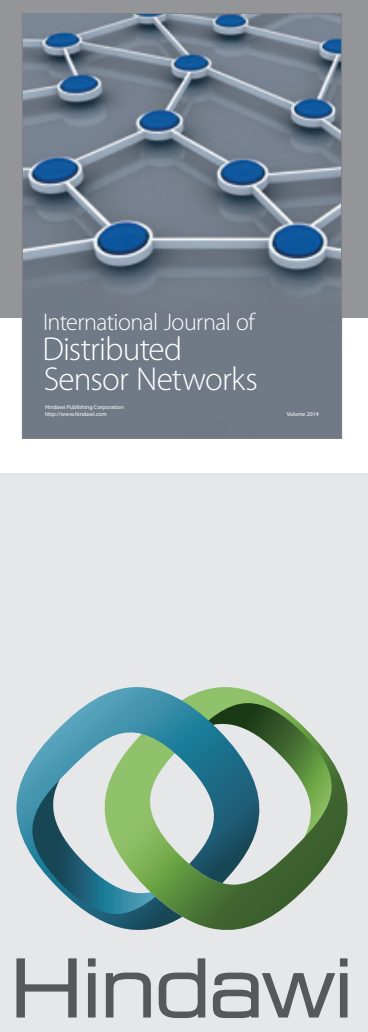

Submit your manuscripts at

http://www.hindawi.com
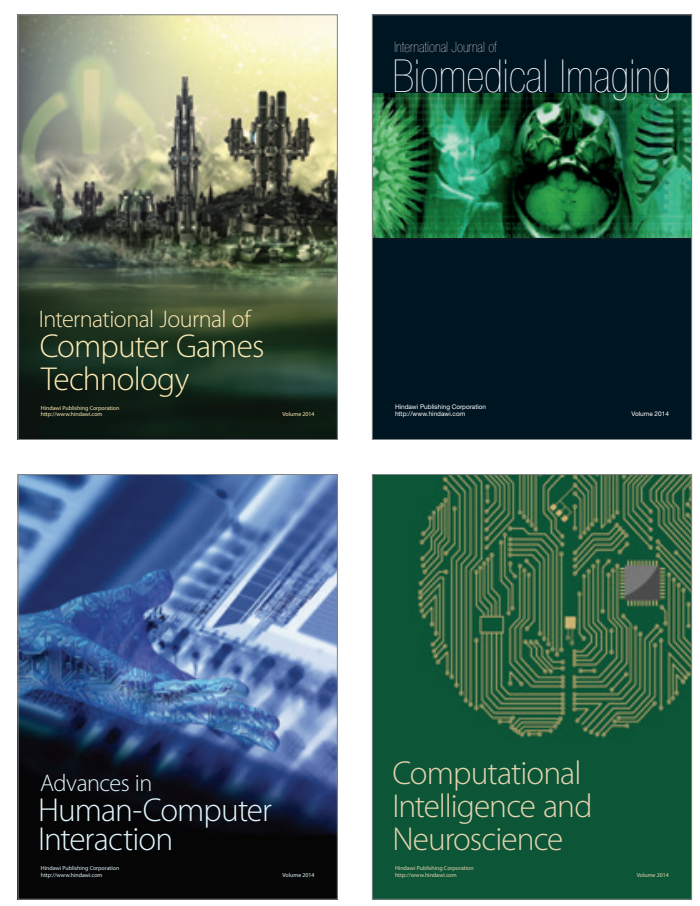
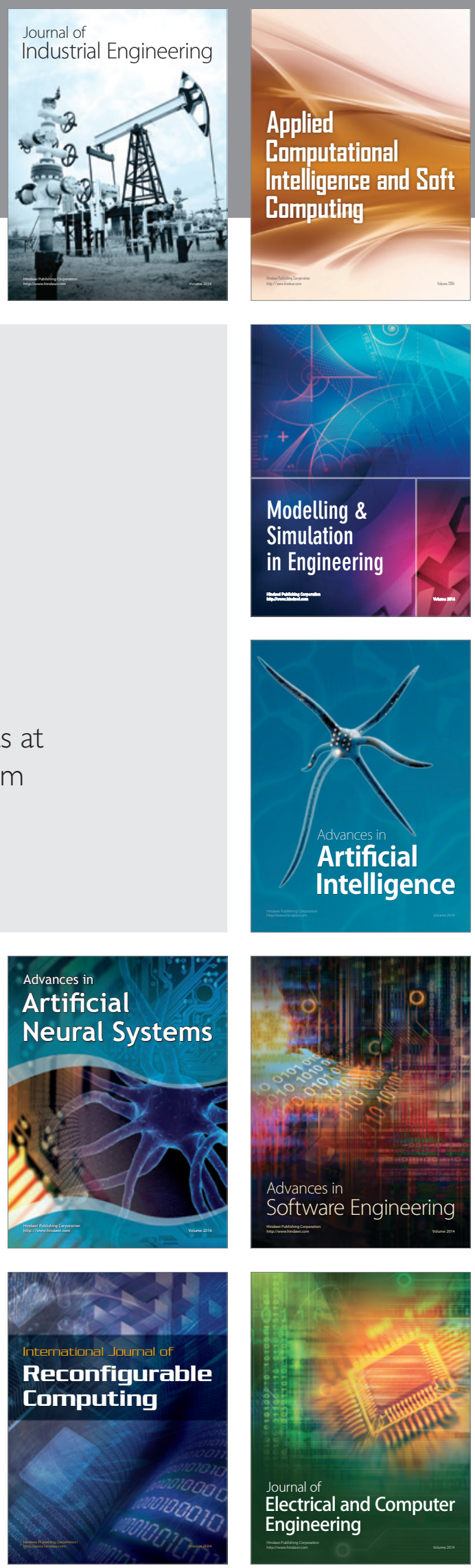